\title{
Plant host selectivity for multiplication of Glomus mosseae spore
}

\begin{abstract}
The study aimed to select plant host for multiplication of Glomus mosseae spores. Five plant species were used [(corn, (Zea mays) sorghum, (Sorghum bicolor) lentil, (Lens culinaris), barley, (Hordeum vulgare) and green bean, (Phaseolus vulgaris)]. Plants were inoculated with Glomus mosseae and grown for 75 days under glasshouse conditions. Mycorrhizal sporulation and colonization of all plant hosts were assessed at different sampling periods. At 75 days of growth the highest number of Glomus mosseae spores was found in mycorrhizosphere of corn plant (167 spore/10 g soil), while the lowest in the mycorrhizosphere of barley (35 spore/10 g soil). The highest percentage of root colonization was in corn (76\%), while the lowest colonization was found in green bean (24\%). Corn was the most suitable host for spore production of Glomus mosseae and to extensive root colonization. It was recorded that plants having more colonization percentage were able to produce more Glomus mosseae spores. The study indicated that different plant species significantly influenced the root spore production and root colonization percentage of Glomus mosseae.
\end{abstract}

Keyword: Glomus mosseae; Host; Root colonization; Spore number 